\title{
MT-AODV: Provendo Resiliência em Redes Ad-Hoc Móveis Militares através de Múltiplas Tabelas de Roteamento sob Demanda
}

\author{
Ricardo M. Firmino, Diogo M. F. Mattos e Dianne S. V. Medeiros
}

Resumo-As Redes Móveis Ad-Hoc (Mobile Ad-Hoc Networks - MANETs) beneficiam aplicações em cenários militares, pois permitem monitorar os sinais vitais dos militares em operações de campo. A comunicação intermitente e a volatilidade de rotas tornam a transmissão de dados desafiadora em MANETs. Desconexões frequentes e consequentes falhas geram atrasos significativos e erros na entrega de mensagens. Este artigo propõe o protocolo Multi-Table Ad-Hoc On-Demand Distance Vector (MTAODV), uma otimização do protocolo de roteamento AODV, para aumentar o desempenho da comunicação em cenários de operações militares com mobilidade de tropas. O MT-AODV calcula múltiplas tabelas de rotas para encontrar múltiplos caminhos possíveis na rede para fornecer maior resiliência e menor latência na comunicação. O desempenho do MT-AODV é comparado ao de outros protocolos tradicionais de MANETs, através de simulação em cenários distintos, com mobilidade e com falha de nós. Os resultados mostram que o MT-AODV apresenta melhor desempenho se comparado ao protocolo original devido ao aumento da disponibilidade de rotas em suas tabelas.

Palavras-Chave-Redes Ad-hoc, protocolos de roteamento, redes sem-fio, tabelas de roteamento

Abstract-The Mobile Ad-Hoc Networks (MANETs) benefit applications in military scenarios, as they enable the monitoring of soldiers' vital signals in field operations. Intermittent communication and route volatility make data transmission in MANETs challenging. Frequent disconnections and consequent failures generate significant delays and message delivery errors. We propose the Multi-Table Ad-Hoc On-Demand Distance Vector (MT-AODV) protocol, an optimization of the AODV routing protocol, to improve communication performance in military operations scenarios with troop mobility. MT-AODV calculates multiple routing tables to find multiple possible paths aiming to provide greater resilience and less latency communication. The MT-AODV performance evaluation was compared to other traditional MANET routing protocols, in a variety of simulated scenarios, with node failure and mobility. The results show that MT-AODV perform better to the original protocol due to the increased availability of paths in its route tables.

Keywords-Ad-hoc networks, routing protocol, wireless networks, routing tables

\section{INTRODUÇÃO}

O monitoramento de combatentes em operações militares fornece previsões sobre a saúde e o desempenho de um indivíduo a partir de seu estado fisiológico em tempo real [1].

Ricardo M. Firmino, Diogo M. F. Mattos e Dianne S. V. Medeiros, Departamento de Engenharia de Telecomunicações, Universidade Federal Fluminense, Niterói-RJ, e-mail: ricardomf@id.uff.br; menezes@midiacom.uff.br; diannescherly@id.uff.br. Este trabalho foi parcialmente financiado por CNPq, CAPES, FAPERJ, FAPESP (2018/23062-5), Marinha do Brasil, Prefeitura Municipal de Niterói/FEC/UFF (PDPA 2020) and RNP.
Essas informações permitem que uma Central de Comando e Controle (CCC) tome decisões para melhorar o desempenho da missão e a capacidade de sobrevivência dos combatentes. Devido à autonomia das Redes Móveis Ad-Hoc (Mobile AdHoc Networks - MANETs) em relação à necessidade de implantação de uma infraestrutura, há uma tendência de aplicar esse tipo de rede no ambiente de operação militar para monitoramento de saúde [2]. No entanto, a comunicação em MANETs é desafiadora devido à complexidade do ambiente de propagação e à mobilidade constante dos nós na rede, sendo necessário adaptar os protocolos a cenários dinâmicos.

Os protocolos de roteamento afetam diretamente o desempenho das aplicações devido à disponibilidade de rotas que influencia a vazão, a taxa de entrega e o atraso dos pacotes transmitidos [3]. A escolha do melhor caminho e a manutenção de rotas são exemplos de desafios que os protocolos de roteamento ad-hoc superam de maneiras diferentes em redes sujeitas a falhas de enlaces ou de nós. Assim, os protocolos atuais buscam melhorar a eficiência da redes de acordo com suas particularidades. O uso de protocolos com múltiplos caminhos pode melhorar o desempenho das aplicações em redes dinâmicas, uma vez que a disponibilidade de rotas é ampliada em comparação ao uso de protocolos com caminhos únicos. Ademais, é possível atingir uma melhor distribuição do tráfego. Contudo, a complexidade e gerenciamento de múltiplas rotas impõem desafios adicionais.

Este artigo propõe o Multi-Tables Ad-hoc On-demand Distance Vector, um protocolo de roteamento que evolui o protocolo AODV, mantendo a retrocompatibilidade. O MT-AODV constrói múltiplas tabelas de roteamento para gerenciamento de múltiplas rotas a fim de aumentar a disponibilidade da comunicação entre nós em uma rede propensa a falha em nós. A ideia é permitir que uma rota alternativa esteja prontamente disponível quando uma falha em nó é detectada. As tabelas de roteamento são criadas sob demanda e as rotas registradas podem ser mais custosas do que o caminho de menor custo. Além de aumentar a disponibilidade da comunicação, é possível aumentar a vazão da comunicação ao utilizar as múltiplas rotas simultaneamente. A implementação da proposta é feita no Network Simulator 3 (NS-3) e a avaliação de desempenho do protocolo é feita através de simulações de diversos cenários, com mobilidade e falhas únicas ou múltiplas de nós. $\mathrm{O}$ desempenho do protocolo é comparado ao de outros protocolos de roteamento tradicionais em MANETs.

Os protocolos de roteamento tradicionais em MANETs computam apenas os caminhos mais curtos, utilizando um 
único caminho para transferência dos dados entre um par origem-destino. Embora algumas adaptações tenham sido realizadas para descoberta de múltiplos caminhos [4], [5], o encaminhamento das mensagens por um caminho alternativo é realizado apenas após falha no caminho principal. Além disso, normalmente as propostas adaptadas de protocolos tradicionais modificam mensagens, tornando os protocolos adaptados incompatíveis com o protocolo original. Diferentemente, este artigo propõe utilizar as mensagens originais e gerenciar múltiplas tabelas de roteamento para seus destinos.

O restante do artigo está organizado conforme a seguir. A Seção 2 apresenta os trabalhos relacionados. A Seção 3 descreve o problema de roteamento multicaminhos para monitoramento de dados de saúde. A Seção 4 apresenta o MTAODV. A Seção 5 descreve os cenários de simulação e discute os resultados obtidos. As conclusões do artigo e os trabalhos futuros são apresentados na Seção 6.

\section{TRABALhos RELACIONADOS}

Os protocolos Destination-Sequenced Distance-Vector (DSDV), Dynamic Source Routing (DSR) ${ }^{1}$, Ad-hoc OnDemand Distance Vector (AODV) ${ }^{2}$ e Optimized Link State Routing $(\mathrm{OLSR})^{3}$ foram desenvolvidos para atender às particularidades das MANETs, e são considerados protocolos de referência. Por este motivo, são usados para comparação na maioria dos trabalhos de pesquisa na área. Diversos trabalhos aproveitam o funcionamento dos protocolos de referência, e os otimizam para obter os benefícios do roteamento por multicaminhos em MANETs, como o MP-DSR [6], AOMDV [4], MAOMDV [5] e MP-OLSR [7]. Contudo, nenhum desses protocolos sugere a distribuição de carga por diferentes caminhos.

As pesquisas para as aplicações de monitoramento de saúde visam estabelecer comunicação entre os sensores de uma mesma rede corporal sem fio. Alguns trabalhos afirmam ainda que, conceitualmente, devido às restrições de uma rede corporal, protocolos de roteamento desenvolvidos para redes ad-hoc comuns não são eficientes para redes corporais [8]. Assim, ainda existe oportunidade de pesquisa para desenvolver novos protocolos de roteamento que foquem em solucionar os desafios encontrados nas redes corporais. Nesse sentido, são propostos na literatura protocolos baseados em cluster, em qualidade de serviço (QoS) [9], em movimentação corporal [10], em redução de temperatura [11], em redução de consumo de energia [12] e cross-layered [13]. No entanto, nenhum desses trabalhos considera o envio de tráfego por múltiplos caminhos. Outros trabalhos [14], [15] provaram, por meio de avaliações de desempenho, que é possível a utilização de protocolos já existentes para MANETs em redes corporais.

Exclusivamente para multicaminhos em monitoramento de saúde, Tseng [16] propõe o Load-Balanced Multipath Routing (LBMR), um protocolo baseado na carga estimada dos nós. O principal objetivo é evitar gargalos de tráfego na rede. Os

\footnotetext{
${ }^{1} \mathrm{~A}$ RFC 4728 define o funcionamento do DSR.

${ }^{2}$ A RFC 3561 estabelece os detalhes para o uso do AODV.

${ }^{3}$ A RFC 3626 especifica a primeira versão do protocolo OLSR. A versão 2 do OLSR é descrita na RFC 7181.
}

resultados mostram melhor desempenho em comparação ao AODV e AOMDV. Birgani et al. [17] apresentam o Reliable Multi-path Routing (RMPR) que utiliza a camada física da rede para construção das rotas.

Diferentemente desses protocolos, o MT-AODV estabelece comunicação por multicaminhos entre diferentes redes corporais, distribuindo a carga. Ao utilizar as mensagens originais do AODV, mantém a retrocompatibilidade. Além disso, não necessita de gerenciamento em camadas como o RMPR.

\section{O PRoblema de Roteamento Multicaminhos para Monitoramento de Dados de Saúde}

Os protocolos de roteamento tradicionais de MANETs buscam os menores caminhos entre os nós e transmitem os dados por um único caminho entre esses nós. Contudo, devido à natureza dinâmica e imprevisível das MANETs, a utilização de múltiplos caminhos é útil [18]. Em operações militares, MANETs são empregadas devido ao ambiente desprovido de infraestrutura. Os combatentes carregam consigo sensores e equipamentos computacionais, favorecendo a tomada de decisão durante a operação [2]. O monitoramento de dados de saúde de múltiplos indivíduos é uma aplicação de tempo real que produz um fluxo contínuo de dados, mas não necessitam de alta taxa de transmissão. É imperativo, no entanto, que o atraso fim-afim seja mínimo. Para permitir a mobilidade dos indivíduos monitorados, a comunicação deve ser sem fio.

A propagação do sinal no ambiente sem fio é complexa devido aos diversos mecanismos de propagação que afetam o sinal. Obstáculos no ambiente e a constante movimentação dos indivíduos levam a falhas nas rotas. Assim, torna-se atrativo o uso de protocolos de roteamento que apresentem maior tolerância a falhas. A utilização de múltiplos caminhos é vantajosa para o desempenho de aplicações de monitoramento de dados de saúde e tende a melhorar a resiliência e a vazão da comunicação. Também afeta positivamente o atraso na entrega de pacotes [19] porque mensagens redundantes podem ser enviadas por diferentes caminhos e, em caso de falha de um caminho, a mensagem poderá ser recebida com sucesso através do caminho alternativo. No entanto, a redundância de mensagens causa sobrecarga na rede. Por isso, muitos protocolos de roteamento buscam múltiplas rotas, mas não operam com a redundância de mensagens. Nesse caso, o fluxo de dados é dividido entre os diferentes caminhos.

As características positivas do roteamento por múltiplos caminhos em relação à tolerância a falhas e à redução do atraso são decorrentes da identificação de rotas alternativas durante a fase de descoberta de rotas. Uma falha no caminho principal resulta na computação de uma nova rota por um protocolo de roteamento de caminho único antes de enviar os novos dados. No protocolo de roteamento por múltiplos caminhos, apenas a fração dos dados transmitidos pelo caminho principal é perdida e novos dados são automaticamente redirecionados para o caminho alternativo ativo sem a necessidade de computar novas rotas, o que reduz o atraso fim-a-fim. Contudo, comparado ao uso de uma rota única ativa e sem falha, o uso simultâneo de múltiplos caminhos pode elevar o tempo para recebimento de todos os pacotes de um fluxo, uma vez que nem todos os caminhos usados são os de menor custo. 


\section{O RoteAmento Ad-Hoc POR Multi-Tabelas}

Este artigo propõe o MT-AODV, que consiste em um protocolo de roteamento capaz de encaminhar os dados por múltiplos caminhos gerenciados por múltiplas tabelas. O MTAODV se baseia no AODV, buscando rotas sob demanda. Enquanto o AODV opera apenas com uma rota para cada destino conhecido, o MT-AODV permite diferentes rotas para o mesmo destino, mas mantém exatamente as mesmas mensagens de controle do AODV, sendo, portanto, retrocompatível. O tráfego origem-destino é dividido e encaminhado pelos diferentes caminhos de maneira uniformemente distribuída. O MT-AODV visa melhorar o desempenho da transmissão dos dados, reduzindo a perda de pacotes no caso de falhas de nós. O MT-AODV é, ainda, um protocolo parametrizável, que possibilita limitar a quantidade de tabelas configuradas, assim como a quantidade de rotas a serem consideradas. Para evitar laços (loops) após a convergência das tabelas de roteamento, os nós intermediários são impedidos de encaminhar de volta para um nó $S$ os pacotes de dados originados em $S$.

O MT-AODV cria múltiplas tabelas sob demanda, à medida em que encontra as rotas. O Algoritmo 1 mostra o procedimento implementado para incluir e atualizar rotas nas tabelas de roteamento quando uma mensagem Route Reply (RREP) é recebida. Sempre que um nó $S$ tem dados para transmitir para um nó $D$ para o qual ainda não possui rotas registradas na tabela, o nó $S$ difunde uma mensagem Route Request ( $R R E Q$ ). O RREQ se propaga através dos nós vizinhos e outros nós intermediários, até chegar ao nó $D$ ou um nó intermediário $K$ que conhece o caminho para $D$. Seja $D$ ou $K$, ao receber a mensagem $R R E Q$ origina-se uma mensagem de resposta RREP transmitida para a origem da requisição, $S$ em modo unicast. Ao receber a mensagem RREP, a função RecvRouteReply é ativada para tratar a mensagem. O resultado final da execução da função é a inclusão ou a atualização da rota encontrada nas tabelas de roteamento de $S$. Cada registro de rota em uma tabela deve conter o destino, o próximo salto (gateway), o custo da rota e o tempo de expiração da rota. Esses parâmetros são extraídos da mensagem usando a função ExtrairInfoRota (linha 5). Destaca-se que o custo da rota neste artigo é calculado em números de salto. Ao receber uma mensagem RREP e extrair as informações sobre a rota, $S$ deve verificar se a rota já está presente em uma de suas tabelas. Isso é feito através da função VerificarRota (linha 6) que percorre as tabelas existentes. Caso exista uma rota com o mesmo gateway, a rota é atualizada na tabela na qual foi encontrada (linhas 9 e 10). Se $S$ possuir uma rota para o mesmo destino $D$, mas com um gateway diferente, a rota é registrada na tabela de roteamento subsequente, de forma que cada tabela possuirá uma rota distinta para o mesmo destino (linhas 14 e 15). Se porventura não houver uma tabela sem uma rota para aquele destino, uma nova tabela de roteamento será criada e sua rota adicionada em seguida (linhas 19 e 20).

Uma vez que o nó de origem pode possuir mais de uma rota para o destino, é possível estabelecer a distribuição do tráfego entre os caminhos existentes. Neste trabalho, a distribuição é feita de forma uniforme entre os $n$ melhores caminhos
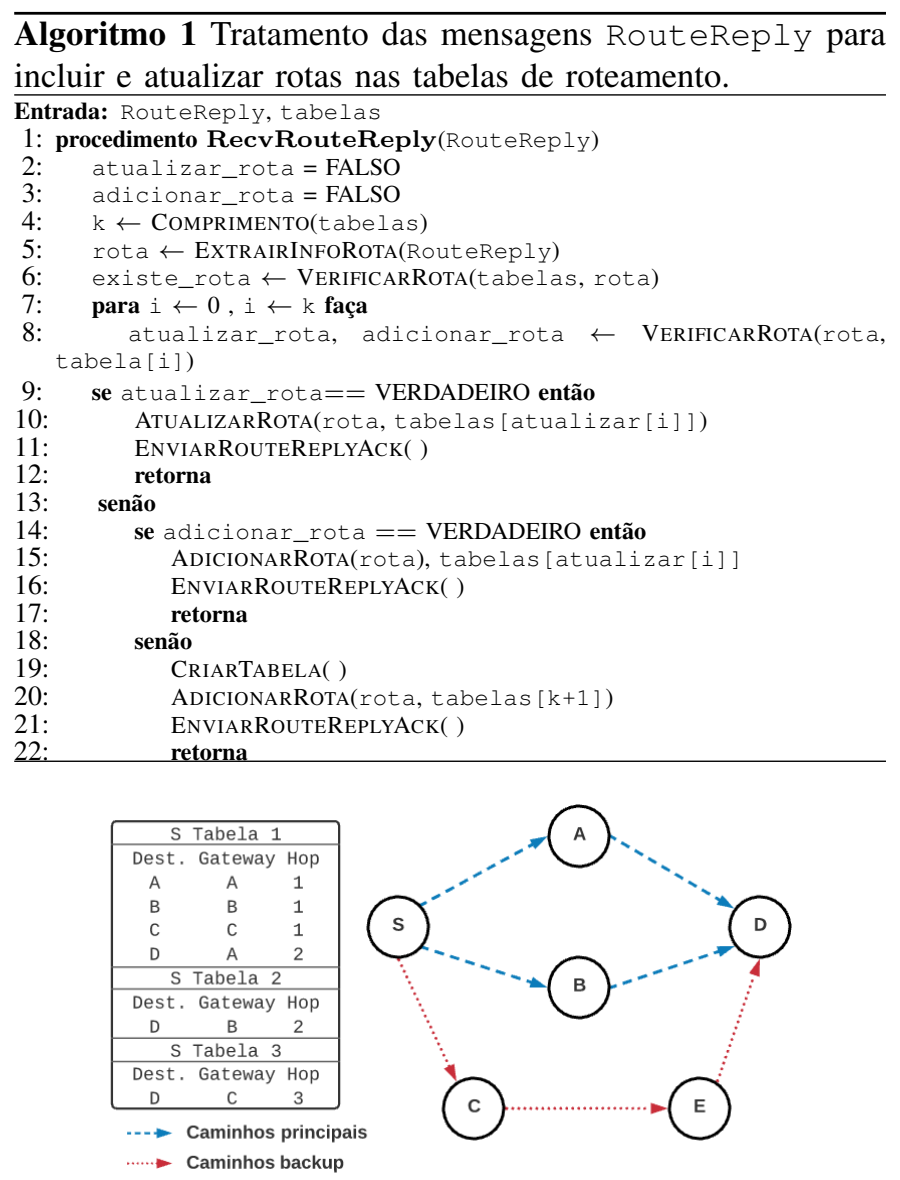

Fig. 1. Topologia hipotética que exemplifica a configuração das tabelas de roteamento do nó de origem $S$. Nas tabelas de roteamento 2 e 3, são mostradas apenas as rotas para o nó destino $D$. Existem dois caminhos principais e um caminho alternativo para salvaguarda registrados nas tabelas consolidadas.

existentes. Dessa forma, os dados são transmitidos entre os caminhos que possuem o menor número de saltos. As demais rotas encontradas atuam como caminhos alternativos para salvaguarda (backup) em caso de falha das rotas principais. A Figura 1 exemplifica as tabelas de roteamento criadas para um nó em uma topologia hipotética e como ocorre a distribuição das mensagens. As rotas para o destino são inseridas a partir do recebimento das mensagens $R R E P$. As tabelas são criadas sob demanda a medida que uma nova rota precise ser inserida.

\section{A Simulação e os Resultados}

O MT-AODV é avaliado através de uma análise comparativa dos resultados obtidos em simulações realizadas para 7 cenários distintos, nos quais variam-se a mobilidade dos nós e o tipo e o número de falhas. As falhas podem ser permanentes ou intermitentes e ocorrem em momentos arbitrados em tempo de execução. Na falha intermitente, o nó permanece em falha durante 5 segundos, retornando ao funcionamento normal após esse período. O nó falhado é escolhido de forma aleatória. $\mathrm{Na}$ falha permanente, escolhe-se aleatoriamente um, dois, três ou quatro nós para falharem a partir de um determinado momento, até o final da simulação. Quando há mobilidade, todos os nós se movem aleatoriamente em uma área. Assim, os cenários são organizados da seguinte forma: 5 cenários com nós fixos (i) uma falha, (ii) duas falhas, (iii) três falhas, (iv) quatro falhas 
e (v) falhas intermitentes em todos os nós; e 2 cenários com mobilidade e (vi) sem falhas e (vii) com falhas intermitentes.

A comparação do MT-AODV é feita com os protocolos DSDV, OLSR e AODV. Para tanto, utiliza-se o Network Simulator 3 (NS-3) versão $3.30^{4}$, um simulador de redes amplamente empregado em pesquisas acadêmicas, desenvolvido em $\mathrm{C}++$. O cenário simulado é composto por uma rede de 9 nós transmissores sem fio e 1 nó coletor de dados, sendo que cada nó representa um dispositivo de monitoramento colocado em um combatente que está em uma operação de campo. Todos os nós são alocados aleatoriamente em um plano de $900 \mathrm{~m}^{2}$. A ideia é que 9 dos dispositivos enviem as informações dos sinais vitais dos respectivos combatentes para o dispositivo em um $10^{\circ}$ combatente que, além de monitorar os sinais vitais também atua como nó coletor. A transmissão dos dados até o nó coletor é feita usando a tecnologia IEEE $802.11 \mathrm{~g}$ e, a partir desse nó os dados são enviados para um Centro de Comando e Controle (CCC). O cenário simulado foca na transmissão até o nó coletor. A transmissão dos 9 nós é realizada simultaneamente com uma pequena diferença aleatória de tempo inserida na simulação para reduzir as colisões no início da simulação. Os nós transmissores geram um tráfego de Taxa de Bits Constante (Constant Bit-Rate - CBR) de $50 \mathrm{~kb} / \mathrm{s}$ para o nó coletor, sendo a carga útil do pacote igual a 256 bytes. Esses valores são adequados para representar a transmissão de informação sobre sinais vitais. Cada execução da simulação dura 120 segundos e são feitas 30 execuções.

A proposta é avaliada quanto ao número total de pacotes recebidos, à indisponibilidade de caminho, ao atraso fim a fim, e à vazão da rede ou taxa de transferência. O número total de pacotes recebidos é o somatório do número de pacotes enviados por cada nó transmissor que é recebido pelo coletor. A indisponibilidade de caminho representa a razão entre o total de pacotes perdidos e o total de pacotes esperados pelo destino, i.e., a relação de uso da rota com o tráfego perdido. $\mathrm{O}$ atraso fim-a-fim mede o tempo médio que cada pacote leva para chegar ao coletor. Por fim, a vazão mostra a média da quantidade de bits recebidos por segundo no nó coletor. Os resultados são médias apresentadas com intervalo de confiança de $95 \%$ considerando a distribuição $t$ de Student.

A Figura 2 apresenta o total de pacotes recebidos pelo nó coletor. Nos cenários com 1 falha e com 4 falhas, o DSDV e o OLSR apresentam melhor desempenho. O MTAODV supera os demais protocolos nos cenários de falha permanente em 3 nós, mobilidade e mobilidade com falhas. Para 2 falhas o MT-AODV supera o AODV mas empata com o OLSR e, no cenário com falha intermitente, o MT-AODV tem desempenho estatisticamente equivalente ao AODV e ao OLSR. A Figura 3 apresenta a indisponibilidade de caminhos. O MT-AODV supera o AODV na maioria das condições e empata nas condições de falhas intermitentes. Esse resultado é consequência da descoberta de mais de uma rota para encaminhamento das mensagens. Assim, consegue-se aumentar a probabilidade de haver um caminho disponível. O MT-AODV desempenha melhor que protocolos proativos nas condições de mobilidade, em que a topologia da rede sofre maiores alte-

${ }^{4}$ https: //www.nsnam.org/releases/ns-3-30/

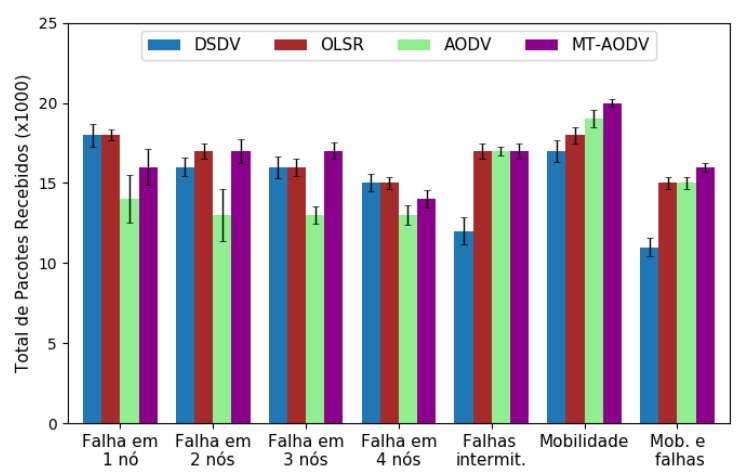

Fig. 2. Número de pacotes recebidos. O MT-AODV supera os demais protocolos nos cenários com 3 falhas, mobilidade e mobilidade com falhas.

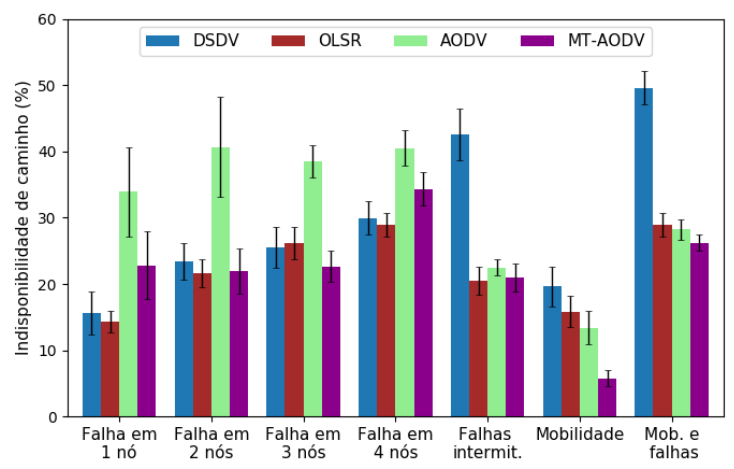

Fig. 3. Percentual de indisponibilidade de caminho. MT-AODV obteve melhor desempenho nas condições de falhas permanente e mobilidade. Nas demais condições, o resultado foi equivalente ao AODV.

rações, dificultando a convergência dos protocolos proativos.

A Figura 4 mostra o atraso fim-a-fim médio dos pacotes recebidos pelo nó coletor. Apesar de o MT-AODV despender menos tempo calculando uma nova rota, o encaminhamento por mais de um caminho aumenta o atraso, uma vez que percursos diferentes podem usar rotas de maior custo. No entanto, comparado ao AODV, o protocolo proposto tem melhor desempenho nos cenário com mobilidade e com falha em 4 nós e é estatisticamente equivalente nos demais cenários. Isso ocorre devido ao grande número de recuperações de rota necessário em condições mais dinâmicas da rede. $\mathrm{O}$ protocolo OLSR apresenta melhores resultados que os demais protocolos. Esse resultado é esperado porque o OLSR é um protocolo proativo, que mantém a topologia da rede sempre atualizada, com rotas disponíveis sempre que elas existirem, não sendo necessário calcular rotas no momento do envio do pacote. Além disso, por ser um protocolo de estado de enlace, o OLSR detecta as falhas rapidamente. Dessa forma, o atraso fim-a-fim tende a ser menor do que o de protocolos reativos. Mesmo sendo reativo e baseado em vetor de distância, o MT-AODV mantém um atraso fim-a-fim estatisticamente equivalente ou muito próximo ao do OLSR na maioria dos cenários, exceto quando há falhas intermitentes. Nesses casos, os protocolos sob demanda procuram uma nova rota ao perceberem a falha, provavelmente de maior custo, mas não retornam para a rota original após término da falha até que a rota atual expire.

A vazão média da rede é apresentada na Figura 5. O resultado mostra que não há queda significativa de desempenho do MT-AODV na maioria dos cenários, mesmo utilizando mais 


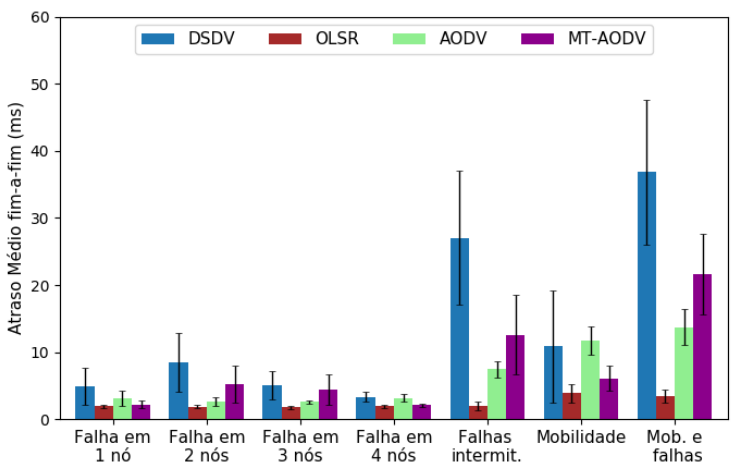

Fig. 4. Atraso fim-a-fim. O MT-AODV obtém melhor desempenho que o AODV nos cenários de falha em 4 nós e de mobilidade. O OLSR apresenta os melhores resultados, por ser um protocolo proativo e de estado de enlace.

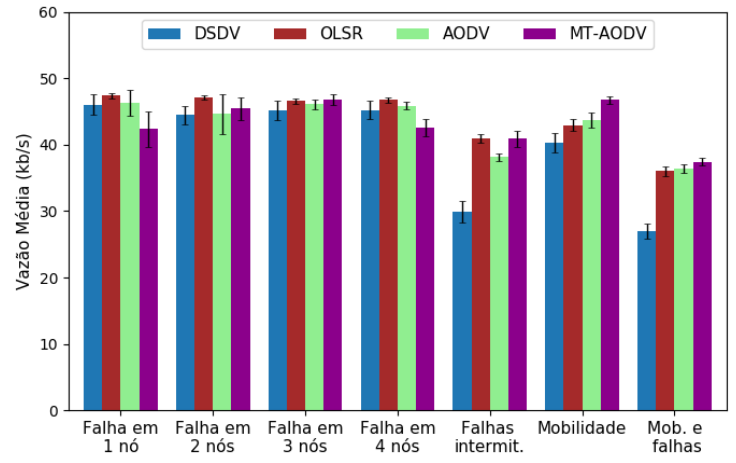

Fig. 5. Vazão média da rede. MT-AODV supera o AODV nos cenários de falhas intermitentes e mobilidade.

de um caminho para transferência dos dados, que aumenta ligeiramente o atraso médio fim-a-fim na maioria dos cenários. No cenário de mobilidade, o MT-AODV alcança maior vazão comparado aos demais protocolos. Comparado ao AODV, o MT-AODV possui resultado superior nos cenários de falhas intermitentes e mobilidade e resultado equivalente nos demais. Os resultados obtidos mostram que o MT-AODV é vantajoso em diferentes métrica e condições, tendo destaque nos cenários de mobilidade, comuns em operações militares em campo.

\section{CONCLUSÃO}

O uso de protocolos de roteamento multicaminhos em MANETs para aplicações de monitoramento de saúde de militares em operações contribui para aumentar a resiliência da rede em locais desprovidos de infraestrutura. Este artigo propôs o MT-AODV, um protocolo reativo resiliente, no qual as rotas são gerenciadas por meio de múltiplas tabelas de roteamento. A proposta é avaliada através de simulações que representam diversos cenários com e sem mobilidade, com tipos e quantidade de falhas variáveis. Os resultados mostram que o MT-AODV alcança melhores resultados em cenários simulados, com destaque para cenários em que há mobilidade. Na condição de mobilidade sem falhas, o MT-AODV alcança aproximadamente $10 \%$ a mais do total de pacotes recebidos e 50\% menos de indisponibilidade de caminho se comparado aos demais protocolos. Vislumbra-se como trabalhos futuros verificar o comportamento do protocolo em aplicações com alta taxa de transmissão, avaliar o desempenho em redes mais densas e verificar o impacto na qualidade da comunicação ao variar o número de caminhos.

\section{REFERÊNCIAS}

[1] K. E. Friedl, "Military applications of soldier physiological monitoring," Journal of Science and Medicine in Sport, vol. 21, no. 11, pp. 11471153, 2018, 4th International Congress on Soldiers' Physical Performance.

[2] W. Harion, K. E. Friedl, M. J. Buller, N. Arango, and R. Hoyt "Evolution of physiological status monitoring for ambulatory military applications," in Human Performance Optimization: The Science and Ethics of Enhancing Human Capabilities. Oxford Univ. Press, 2018, p. 142 .

[3] D. M. F. Mattos, L. H. G. Ferraz, L. H. M. K. Costa, and O. C M. B. Duarte, "Evaluating virtual router performance for a pluralist future internet," in Proceedings of the 3rd International Conference on Information and Communication Systems, ser. ICICS '12. New York, NY, USA: Association for Computing Machinery, 2012.

[4] M. K. Marina and S. R. Das, "On-demand multipath distance vector routing in ad hoc networks," in Proceedings Ninth International Conference on Network Protocols. ICNP 2001. IEEE, 2001, pp. 14-23.

[5] V. Matre and R. Karandikar, "Multipath routing protocol for mobile adhoc networks," in 2016 Symposium on Colossal Data Analysis and Networking (CDAN), 2016, pp. 1-5.

[6] A. Nasipuri and S. R. Das, "On-demand multipath routing for mobile ad hoc networks," in Proceedings Eight International Conference on Computer Communications and Networks (Cat. No. 99EX370). IEEE, 1999, pp. 64-70.

[7] J. Yi, A. Adnane, S. David, and B. Parrein, "Multipath optimized link state routing for mobile ad hoc networks," Ad hoc networks, vol. 9 , no. 1, pp. 28-47, 2011.

[8] S. Movassaghi, M. Abolhasan, J. Lipman, D. Smith, and A. Jamalipour, "Wireless Body Area Networks: A Survey," IEEE Communications Surveys Tutorials, vol. 16, no. 3, pp. 1658-1686, 2014.

[9] S. CP et al., "Qos-aware minimum cost routing algorithm for wireless body area networks." Adhoc \& Sensor Wireless Networks, vol. 47, 2020.

[10] A. Maskooki, C. B. Soh, E. Gunawan, and K. S. Low, "Opportunistic routing for body area network," in 2011 IEEE Consumer Communications and Networking Conference (CCNC). IEEE, 2011, pp. 237-241.

[11] E. Rosillo, V. Ferreira, R. Lima, C. Albuquerque, and D. MuchaluatSaade, "Lator: Protocolo de roteamento ciente de qualidade com prevenção de aquecimento para redes corporais sem fio," in Anais do XXXVIII Simpósio Brasileiro de Redes de Computadores e Sistemas Distribuídos. Porto Alegre, RS, Brasil: SBC, 2020, pp. 854-867.

[12] F. Ullah, M. Zahid Khan, M. Faisal, H. U. Rehman, S. Abbas, and F. S. Mubarek, "An energy efficient and reliable routing scheme to enhance the stability period in wireless body area networks," Computer Communications, vol. 165, pp. 20-32, 2021.

[13] Q. Liu, K. G. Mkongwa, C. Zhang, and S. Wang, "A simple cross-layer mechanism for congestion control and performance enhancement in a localized multiple wireless body area networks," Journal of Ambient Intelligence and Humanized Computing, pp. 1-18, 2021.

[14] P. He, X. Li, L. Yan, S. Yang, and B. Zhang, "Performance analysis of wban based on aodv and dsdv routing protocols," in 2015 2nd International Symposium on Future Information and Communication Technologies for Ubiquitous HealthCare (Ubi-HealthTech). IEEE, 2015 , pp. 1-4.

[15] V. Ferreira, F. Seixas, D. Muchaluat-Saade, and C. Albuquerque, "Análise do protocolo aodv para roteamento em wireless body area networks," Anais do VII Simpósio Brasileiro de Engenharia de Sistemas Computacionais (SBESC), pp. 1-8, 2017.

[16] C. H. Tseng, "LBMR: Load-Balanced Multipath Routing for wireless data-intensive transmission in real-time medical monitoring," International journal of environmental research and public health, vol. 13, no. 6, p. 547, 2016.

[17] Y. G. Birgani, N. T. Javan, and M. Tourani, "Mobility enhancement of patients body monitoring based on wban with multipath routing," in 2014 2nd International Conference on Information and Communication Technology (ICoICT), 2014, pp. 127-132.

[18] S. Mueller, R. P. Tsang, and D. Ghosal, "Multipath routing in mobile ad hoc networks: Issues and challenges," in Performance Tools and Applications to Networked Systems, M. C. Calzarossa and E. Gelenbe, Eds. Berlin, Heidelberg: Springer Berlin Heidelberg, 2004, pp. 209_ 234.

[19] J. Tsai and T. Moors, "A review of multipath routing protocols: From wireless ad hoc to mesh networks," in ACoRN early career researcher workshop on wireless multihop networking, vol. 30. Citeseer, 2006. 